\title{
Educating astrometry and celestial mechanics students for the 21st century
}

\author{
W. F. van Altena ${ }^{1}$ and M. Stavinschi ${ }^{2}$ \\ ${ }^{1}$ Yale University, P.O. Box 208101, New Haven, CT 06520, USA \\ email: vanalten@astro.yale.edu \\ ${ }^{2}$ Astronomical Institute of the Romanian Academy, Cutitul de Argint 5, Bucharest, \\ RO-040557, Romania \\ email: magda_stavinschi@yahoo.fr
}

\begin{abstract}
Astrometry and Celestial Mechanics have entered a new era with the advent of Micro-arcsecond positions, parallaxes and proper motions. Cutting-edge science topics will be addressed that were far beyond our grasp only a few years ago. It will be possible to determine definitive distances to Cepheid variables, the center of our Galaxy, the Magellanic Clouds and other Local Group members. We will measure the orbital parameters of dwarf galaxies that are merging with the Milky Way, define the kinematics, dynamics and structure of our Galaxy and search for evidence of the Dark Matter that makes up most of the mass in the universe. Stellar masses will be determined routinely to $1 \%$ accuracy and we will be able to make full orbit solutions and mass determinations for Extrasolar planetary systems. If we are to take advantage of Micro-arcsecond astrometry, we need to reformulate our study of reference frames, systems and the equations of motion in the context of special and general relativity. Methods also need to be developed to statistically analyze our data and calibrate our instruments to levels beyond current standards. As a consequence, our curricula must be drastically revised to meet the needs of students in the 21st Century. With the above considerations in mind, we developed a syllabus for an introductory one-semester course in Astrometry and Celestial Mechanics. This course gives broad introductions to most topics in our fields and a base of knowledge from which a student can elect areas for self-study or attendance at centers where advanced courses, workshops or internships are available.
\end{abstract}

Keywords. astrometry, sociology of astronomy, education

\section{Introduction}

Astrometry and Celestial Mechanics have entered into a new era with the advent of Micro-arcsecond positions, parallaxes and proper motions. Cutting-edge science topics will be addressed that were far beyond our grasp only a few years ago. It will be possible to determine definitive distances to Cepheid variables, the center of our Galaxy, the Magellanic Clouds and other Local Group members. We will measure the orbital parameters of dwarf galaxies that are merging with the Milky Way, define the kinematics, dynamics and structure of our Galaxy and search for evidence of the Dark Matter that makes up most of the mass in the universe. We have already measured the mass of the Black Hole at the center of the Milky Way and will soon be able to determine the detailed mass distribution of the galactic center. Stellar masses will be determined routinely to $1 \%$ accuracy and we will be able to make full orbit solutions and mass determinations for Extrasolar planetary systems.

If we are to take advantage of Micro-arcsecond astrometry, we need to reformulate our study of reference frames, systems and the equations of motion in the context of special and general relativity. The reference frames and systems established by Gaia, an orbiting 
satellite, must be related to those of the Solar System and the Earth, hence we must consider Celestial Mechanics as an integral part of our students educational program. Finally, methods need to be developed to calibrate our instruments to levels beyond commonly expected standards. In addition to the numerous space-based missions that are planned to provide high accuracy positions, parallaxes and proper motions, several large-scale ground-based telescopes and instruments such as Pan-STARRS, LSST and large-scale CCD mosaic camera surveys will dramatically increase the quantity of data available and require that students be educated in techniques to analyze massive amounts of data to cull out trends and special objects. As a consequence, our curricula must be drastically revised to meet the needs of students in the $21^{\text {st }}$ Century.

A full range of courses is available to students at only a few centers in Europe and Asia, so it will be necessary to utilize a variety of teaching methods and opportunities, such as lecture courses, internships, workshops and summer schools. The 2005 Yale Summer School on Astrometry is an example of an intensive introduction to Astrometry. The School web site (http://www.astro.yale.edu/workshop) contains the lectures, which may be freely downloaded. The IAU Division I Working Group on Astrometry by small ground-based telescopes can provide numerous educational and training opportunities for students and a means to accomplish different international campaigns, such as PHEURA, PHESAT and PHEMU.

\section{An introductory course on astrometry and celestial mechanics(ACM1)}

With the above considerations in mind, we developed a syllabus for an introductory 40-hour one-semester course in Astrometry and Celestial Mechanics, which we refer to here as ACM 1. A course length may vary at different institutes, but it should be easy to revise this to a 20-hour course by reducing the depth covered in subjects or by selectively omitting some areas. In addition, the relative stress on subject areas should be adjusted to meet the special needs of different institutions. As noted above, there are very few centers where a full range of courses can be offered, therefore we developed the syllabus for a one-semester course that can be offered at many institutions and provide a maximum number of students with an introduction to Astrometry and Celestial Mechanics. This course gives broad introductions to most topics in our fields and a base of knowledge from which a student can elect areas for self-study or attendance at centers where advanced courses, workshops or internships are available.

Our fields are small and specialized so it is vital that ACM 1 be integrated into a general astronomical curriculum with offerings in fields that are significantly impacted by the application of modern astrometric and celestial mechanical techniques. Such areas include: galactic structure and dynamics, stellar astrophysics, the formation and evolution of planets and stars, as well as introductions to extragalactic astronomy and cosmology. With such a diverse background our students will be able to integrate into the astronomical community and optimally apply astrometric and celestial mechanical techniques to the solution of problems relevant to modern astrophysics. It is important that each subject area be introduced with science applications that will help to motivate students to study the topic.

ACM 1 was developed to meet the first level of educational needs for a Ph.D. in our fields. It will be necessary for each institute to revise the subject areas included in ACM 1 for Masters degree programs where the students are normally expected to be more specialized and to eventually play supporting roles in large scale research programs. 
With the above factors taken into consideration we present here our proposed syllabus for an introduction to the study of Astrometry and Celestial Mechanics and hope that it will be of use to our colleagues in preparing the next generation of scientists in our fields. Each subject area is preceded by the recommended number of hours to be spent on the topic in the context of a 40-hour course.

\section{The Syllabus for ACM 1}

- (1 hr) Introduction and background:

- Hipparcos, Gaia, SIM and other astrometric projects as motivations to study astrometry

- (2 hr) Trigonometric Parallaxes:

- Highlights: Gaia and SIM give parallaxes of Cepheids, RR Lyraes, the Galactic Center and the Magellanic Clouds

- Absolute versus relative parallax

- Calibration of luminosities

- Mass-Luminosity relation

- (2.5 hrs) Binary and multiple stars:

- Highlights: Mass of the Milky Way's Black Hole, ...

- Determining orbital parameters from observations

- Speckle interferometric observations

- Space-based methods and the principal results

- Long baseline optical interferometry (CHARA, VLTI, Keck, PRIMA, ...)

- Surveys of binary parameters

- (0.75 hr) Star clusters:

- Highlights: Gaia and SIM give definitive distances, kinematical masses and membership

- Membership determination

- Internal motion determination

- (1.0 hr) Solar System astrometry:

- Highlights: Finding extra-solar planets, ...

- Moving object astrometry

- NEOs, Minor planets and comets

- Algorithms

- Observing in the glare of a bright planet

- Lunar occultations

- Extra-solar planets

- Optical astrometry

- Radial velocity observations

- Space-based discovery (SIM, Gaia, Kepler, ...)

- (2 hrs) Galactic Structure astrometry:

- Highlights: Gaia and SIM define the structure and dynamics of our Milky Way

- The role that astrometry plays in defining the spatial, kinematical and dynamical structure of the Galaxy and the Local Group of galaxies

- Modifications to the equations required by Micro-arcsecond proper motions

- (1.5 hr) Statistical astronomy:

- Highlights: Modeling and correcting for systematic effects are critical

- Statistical and secular parallaxes 
- The Malmquist and Lutz-Kelker corrections

- Monte-Carlo modeling

- (0.5 hr) Cosmology astrometry:

- Highlights: How astrometry can help even at the edge of the universe

- Constraining critical cosmological parameters.

- Search for primordial gravitational waves

- Astrometry of gamma-ray bursters

- (1 hr) The Earth's atmosphere (troposphere):

- Highlights: How we model the atmosphere

- Models of the atmosphere and turbulence

- Refraction through a turbulent atmosphere

- Refraction and absorption as a function of wavelength

- The effect of refraction on positions

- Special cases for Radio Astronomy

- (1 hr) Atmospheric limits to positional precision (optical and radio):

- Highlights: Beating atmospheric turbulence in large fields of view

- Observational determination of the limits

- Theoretical interpretation of the observations

- Tip-tilt and adaptive optics correction systems

- Image reconstruction

- Phase referencing

- (0.5 hr) Diffraction-limited imaging:

- Highlights: Getting the ultimate resolution from your optics

- HST, Gaia, and Hipparcos

- Speckle imaging

- Ground-based observations in the infra-red

- (2 hrs) Interferometry (Optical):

- Highlights: Beating atmospheric turbulence in small fields of view

- Theory

- Sparse vs filled apertures

- Imaging

- Coronagraphy

- Optical

- Michelson interferometers: theory and calibration

- Existing systems: VLTI, Keck, CHARA, NPOI, ...

- Planned systems: PRIMA, SIM

- Intensity interferometers

- White-light interferometers, such as the HST/FGS

- Speckle Interferometers

- (2 hrs) Interferometry (Radio):

- Highlights: The VLBI micro-arcsecond parallaxes

- http://veraserver.mtk.nao.ac.jp/hilight/pub070711/pub070711-e.html

- Theory and calibration of existing systems

- Connected-element, VLA, VLBI, VLBA, VERA

- Phase referencing

- Physics of water masers

- Gravitational deflection of light 
- Future instruments

- ALMA, SKA

- (3 hrs) Geometrical optics for Astrometry:

- Highlights: The imaging properties of optical telescopes

- Refracting optics

- Filters \& CCD dewar windows

- Beam shift, focus and image position errors

- Seidel aberrations

- Theory

- Aberrations relevant to optical astrometry

- Observational examples

- Reflecting optics

- Conic sections

- Seidel aberrations

- Two-mirror telescopes

- Aberrations

- Alignment

- Astrometric compensation for optical aberrations

\section{- (1.5 hrs) Imaging detectors:}

- Highlights: The complexities of our modern detectors

- Characteristics of astronomical CCD and CMOS detectors

- Calibrating

- The signal sources

- Noise sources (flat, bias, dark and read-out)

- Charge-transfer efficiency (CTE)

- Linearity and saturation

- Fringing

- Optimizing the signal-to-noise

- Astrometry

- Image centroids and PSF

- Photometry

- Surface, Aperture and PSF photometry

- Statistical techniques useful for imaging analyzes

- (0.25 hr) Time-delayed integration:

- Highlights: Letting the sky drift by; observing efficiently

- Gaia, CTI, SDSS, and QuEST.

- (1 hr) Image deconvolution:

- Highlights: Increase your telescope aperture by 40\%, ...

- Theory

- Noise sources

- Aberrated PSFs

- Good PSFs but correcting for atmospheric degradation

- Potential gain in precision

- Potential gain in limiting magnitude

- (1 hr) Plate measuring machines:

- Highlights: Old plates can improve our proper motions

- Scanning machines

- SuperCOSMOS, PDS, STScI GAMMA 
- Calibrating for thermo-mechanical drifts and non-linearities

- Step-and-repeat imaging of a plate

- PMM, StarScan, next generation scanners

- Stitching the "footprints" together

- Algorithms for photographic images

- Astrometry

- Photometry

- Calibration

- Orthogonality of axes, periodic errors, detector time lags, and others

- (3 hrs) From measures to celestial coordinates:

- Highlights: Projecting the sky onto the focal plane

- Primer on spherical astronomy

- Telescope-modeling techniques.

- Gnomonic to focal plane calibrations

- Focal-plane arrays of CCDs

- Looking for systematic errors

- Extending astrometric calibration regions to fainter objects and to different passbands.

- (1 hr) Calibration of complex instrumental systems:

- Highlights: Calibration makes your instrument and data valuable

- Mosaic cameras

- HST Fine Guidance Sensors

- Hipparcos and Gaia

- Time-delayed integration systems

- SDSS, QuEST

- (3 hrs) Relativistic foundations of astrometry and celestial mechanics:

- Basics of special and general relativity

- Post-Newtonian approximation scheme

- Relativistic astronomical reference systems and frames

- Coordinate-dependent and measurable quantities

- Relativistic astronomical time scales and their realizations

- Relativistic data reduction modeling

- Relativistic equations of motion (test body around a spherically symmetric body and N-body problem)

- Relativistic effects needed for micro-arcsecond astrometry

- (3 hrs) Stellar Coordinate Systems and Positions:

- Glossary of terms used and definitions

- Transformation from ICRS to Observed Places of Stars

- Flow charts and formulae for the transformations

- IAU Nomenclature for Fundamental Astronomy — "Explanatory Document B, July 2006" - http://syrte.obspm.fr/iauWGnfa/NFA_B.pdf

\section{- (4 hrs) Celestial Mechanics of the N-body problem:}

- The Sun, solar oblateness

- Major planets, planetary rings

- Minor planets, asteroid belts

- The role of analytic techniques and numerical methods

- The role of pulsar timing, laser and radio ranging

- High-precision radial velocities 
- Analyzing binary and multiple systems with micro-arcsecond precision

- (2 hrs) Catalogs:

- Highlights: Making the observations usable and accessible

- Fundamental

- ICRF and radio astrometry

- Optical reference system

- ICRS, Hipparcos (HCRF) and Tycho

- Looking to Gaia

- Extending to fainter magnitudes

- UCAC, NPM \& SPM

- Value of "non-astrometric" catalogs: 2MASS, DENIS, SDSS

- Schmidt-based catalogs

- GSC, Digital Sky Survey, USNO A2.0 \& B-1, Cosmos, SRC

- The Virtual Observatory

- National versions of the VO

- NOMAD - the "astrometric" VO

\section{- (0.5 hr) Analyzing poorly-sampled images:}

- Highlights: Increasing accuracy and compensating for systematic effects

- HST imaging astrometry and photometry

\section{Conclusion}

The evolution of Astrometry from ground-based to space-based has resulted in greatly increased accuracy that requires us to reformulate our reference frames, systems and equations of motion in the context of special and general relativity. Our old curricula are outdated and need to be completely revised especially in countries that have direct access to space-based observations. A range of teaching methods and opportunities are needed, such as lecture courses, workshops, summer schools and internships. The IAU Division I Working Group on Astrometry by small ground-based telescopes can provide numerous educational and training opportunities for students and a means to accomplish different international campaigns, such as PHEURA, PHESAT and PHEMU.

\section{Acknowledgements}

We are grateful to colleagues who provided us with many suggestions for the onesemester syllabus in Astrometry and Celestial Mechanics: Sergei Kopeikin, Sergei Klioner, Nicole Capitaine, Anita Gomez, Wenjing Jin, Stephen Unwin, Elliott Horch, Stephen Majewski, Michael Efroimsky and others.

We acknowledge NSF, NASA, ESA, ESO and our national observatories all of whom provide us with outstanding facilities for astrometric research. 